\title{
Pulsed NMR in Linear Aliphatic Polyesters
}

\author{
Masayoshi Ito, Hajime Serizawa, and Koji TanaKa \\ Department of Chemistry, Faculty of Science, Science University of Tokyo, \\ Kagurazaka, Shinjuku-ku, Tokyo 162, Japan.
}

(Received September 14, 1977)

\begin{abstract}
Solid echo NMR measurements were carried out for poly(ethylene sebacate) (2-8), poly(decamethylene 1,16-hexadecanedicarboxylate) (10-16), and chemically degraded 2-8 polyesters. The following results were found: (1) all the samples are composed of three phases: i.e., crystalline, intermediate, and amorphous components; (2) the $\gamma$ - and $\beta$ relaxations are found in the amorphous component of the 2-8 and 10-16 polyesters, in agreement with earlier conclusions from dielectric measurements; (3) in the $\beta$-relaxation, the chain mobility in the amorphous region and the value of the activation energy increase with increasing ester group concentration in the polyester; but the degree of restriction imposed upon the local mode motion in the amorphous region is greatly influenced not only by the ester group concentration but also by the morphology of the sample; (4) in the chemically degraded 2-8 polyester, the content of the intermediate phase decreases with increasing crystallinity, however, the mobility of this phase is less sensitive to both crystallinity and temperature.
\end{abstract}

KEY WORDS Pulsed NMR / Linear Aliphatic Polyesters / Solid Echo / Poly(ethylene sebacate) / Poly(decamethylene 1,16-hexadecanedicarboxylate) / Chemically Degraded Polyester / Intermediate Component / Chain Mobility / Ester Group Concentration / Morphology /

Recently, much attention has been focused on the effects of chemical structure on the morphology of semicrystalline polymers such as polyamides ${ }^{1}$ and polyesters. ${ }^{2,3}$ It is of interest to investigate the relation between the chemical structure and the relaxation behavior of these crystalline polymers.

Linear aliphatic polyesters are particularly appropriate for elucidating this relation, since the morphological regularity and simplicity of single crystals of the polyesters have been correlated with chemical structure of the polymer. ${ }^{2,3}$

In a previous paper, ${ }^{4}$ we reported the dielectric relaxation behavior of some linear aliphatic polyesters. In the $\beta$-relaxation which corresponds to the glass transition of the polyesters, the loss peak tempearture and the effective dipole moment of the motional unit are correlated with the chemical structure of the polymer. It is possible that the effect of chemical structure on the molecular motion may also be found in the other relaxation mechanisms of the polyesters.

Pulsed NMR studies of heterogeneous polymer systems have become accepted as one of the most effective techniques for obtaining direct information about the molecular motion of each phase. $^{5,6}$ In this paper, first, the temperature dependence of the spin-spin relaxation times for two polyesters with different ester group concentrations was studied in an attempt to investigate what changes in molecular motion in each phase occur as a result of the differences of chemical structure of the polyesters. Second, the nature of the intermediate phase of the polyester was discussed on the basis of the NMR data of the chemically degraded polyester.

The polyesters used in the present study were poly(ethylene sebacate) and poly(decamethylene 1,16-hexadecanedicarboxylate), hereafter referred to as $2-8$ and $10-16$ polyesters. 


\section{EXPERIMENTAL}

\section{Samples}

The polyesters were synthesized from appropriate glycols and dibasic acid dimethyl esters by ester exchange. Details of the preparation are given in a previous paper. ${ }^{4}$ Bulk crystallized samples (MG) were prepared by hot pressing at above their melting temperature and cooled slowly to room temperature in vacuo.

Solution grown crystals (SG) of the $2-8$ polyester were obtained from a $0.05 \%$ by weight solution in isoamylacetate at $38^{\circ} \mathrm{C}$. After isothermal crystallization for $24 \mathrm{hr}$, the resulting single crystals were dried in vacuo at room temperature for a month.

The physical characteristics of these samples are summarized in Table I.

Table I. Physical characteristics of the samples

\begin{tabular}{crc}
\hline Sample & $M_{\mathrm{n}}$ & $X_{\mathrm{c}}(\%)$ \\
\hline 2-8-MG & 13,000 & 56 \\
2-8-SG & 13,000 & 66 \\
$10-16-\mathrm{MG}$ & 17,000 & 57 \\
\hline
\end{tabular}

2-8 polyester was degraded in the following way: a film about $300-\mu \mathrm{m}$ thick was cast from benzene solution and annealed at $72^{\circ} \mathrm{C}$ for $5 \mathrm{hr}$ in vacuo. The film was degraded for various times in $70 \%$ aqueous mono-ethyl amine at $30^{\circ} \mathrm{C}{ }^{7}$ The film was stirred throughout the reaction. After the reaction, the samples were washed by ethanol several times and then dried in vacuo at room temperature for $50 \mathrm{hr}$.

\section{Measurements}

Plused NMR experiments were carried out with a JEOL pulsed NMR (JSE-5B) spectrometer, operating at a frequency of $60 \mathrm{MHz}$. The solid echo sequence, ${ }^{8} 90^{\circ}-\tau-90^{\circ}\left(90^{\circ}\right.$ phase shift $)$, provided an approximation to the complete free induction decay, from which the spin-spin relaxation time $\left(T_{2}\right)$ was obtained. In this study, $\tau$ and the pulse width have been adjusted to be 9 and $2 \mu \mathrm{sec}$, respectively. Solid echo signals were stored and then read out on the pen recorder, using an Iwatsu DM-701 digitizer. Samples were packed in a sample tube in vacuo. The sample temperature was regulated to $\pm 0.5^{\circ} \mathrm{C}$ by a nitrogen gas flow thermostat.
The melting temperature and heat of fusion of the samples were observed by using a Perkin-Elmer differential scanning calorimeter (DSC-1B) in a nitrogen atomosphere. The crystallinity of the samples was calculated from the observed heat of fusion. 32.4 and $49.1 \mathrm{cal} / \mathrm{g}$ were used as the heats of fusion of $2-8$ and $10-16$ polyesters. 9 The number-average molecular weight was estimated by vapor pressure osmometry and the long spacing was evaluated from small-angle X-ray photographs.

\section{RESULTS AND DISCUSSION}

Some examples of the solid echo decays obtained for the 2-8-MG and 10-16-MG are shown in Figure 1. It is immediately apparent from the photos that the observed decays are not of the single exponential form. Beat signals (not shown in this paper) were observed below about $-80^{\circ} \mathrm{C}$ in every sample. A similar signal for polyethylene and Nylon 6 has been found by Fujimoto, et al., and iterpreted to mean that the absorption curve given by the Fourier transform of the signal is not Gaussian but rather bell-shaped. ${ }^{5}$ In the present study no analysis of the beat signal was carried out.

\section{Decomposition of Decays to Components}

Figure 2 shows the logarithm of the solid echo decays of the $2-8-\mathrm{MG}$ at $25^{\circ} \mathrm{C}$ plotted against time. For times greater than $130 \mu \mathrm{sec}$ the experimental points fall on a single straight line $\left(\mathrm{L}_{1}\right)$ very well. The exponential portion of the curve can be written as ${ }^{10}$

$$
S(t)=S_{0} \exp \left(-t / T_{2}\right)
$$

where $T_{2}$ is called the spin-spin relaxation time. Such an exponential curve is expected if the material is in the motionally narrowed region. To examine the portion of the curve which deviates from the line $\mathrm{L}_{1}, \mathrm{~L}_{1}$ was extrapolated back to time zero and the appropriate value subtracted from the experimental data. For times greater than $30 \mu \mathrm{sec}$, the subtracted points, designated by the symbol ( $\bullet$ ) in Figure 2, also fall on a straight line $\left(\mathrm{L}_{2}\right)$. Further, to examine the initial portion of the curve which deviates from $\mathrm{L}_{2}, \mathrm{~L}_{2}$ was extrapolated back to time zero and the appropriate value subtracted again from the curve. The initial portion of the data treated in this manner is shown 


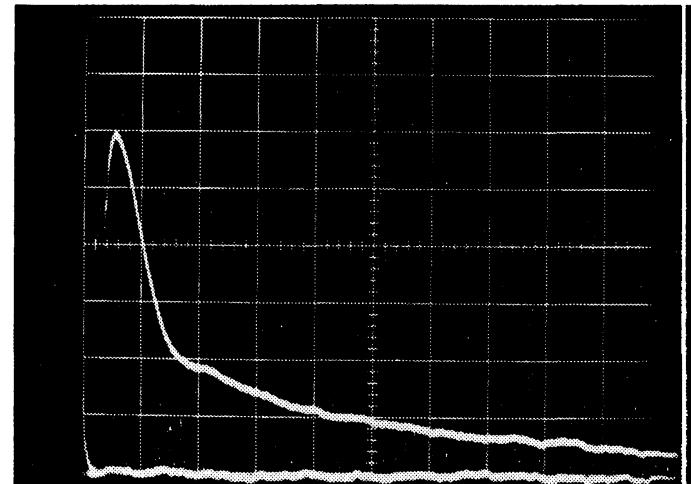

(a)

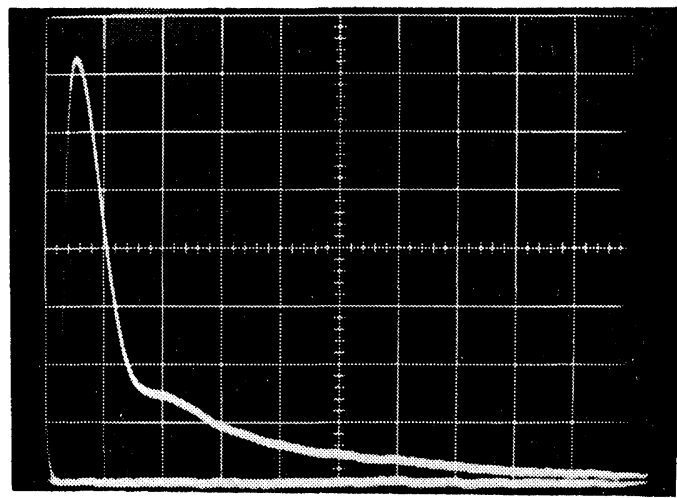

(c)

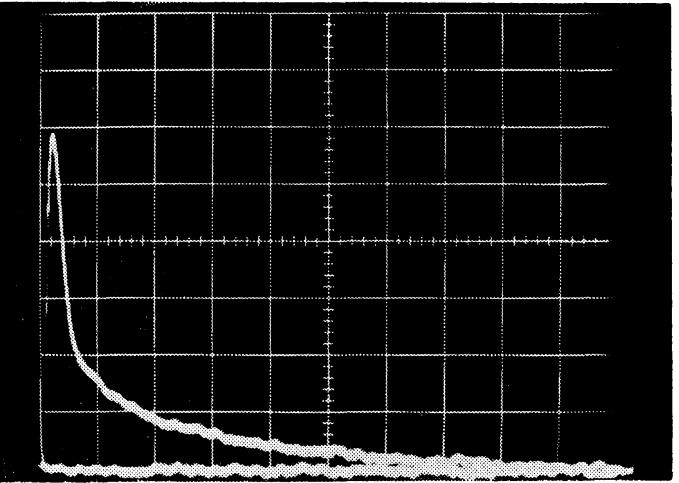

(b)

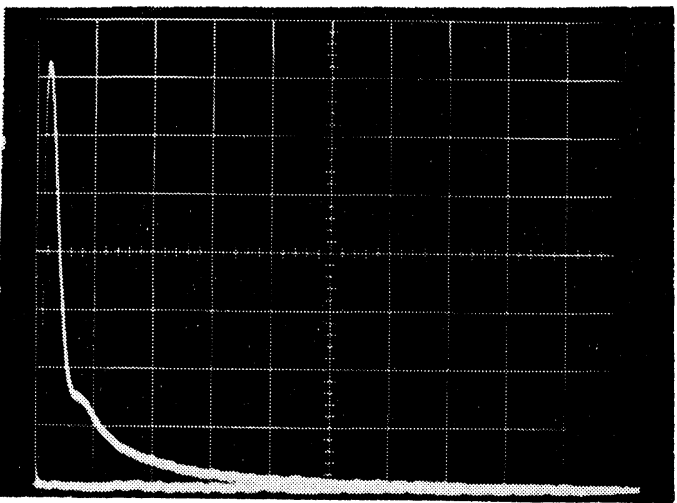

(d)

Figure 1. Solid echo signals from the 2-8-MG and 10-16-MG at $25^{\circ} \mathrm{C}$ : (a) $2-8-\mathrm{MG}, X=20 \mu$ $\mathrm{sec} / \mathrm{unit}$; (b) $2-8-\mathrm{MG}, X=50 \mu \mathrm{sec} / \mathrm{unit}$; (c) 10-16-MG, $X=20 \mu \mathrm{sec} / \mathrm{unit}$; (d) 10-16-MG, $X=50$ $\mu \mathrm{sec} /$ unit.

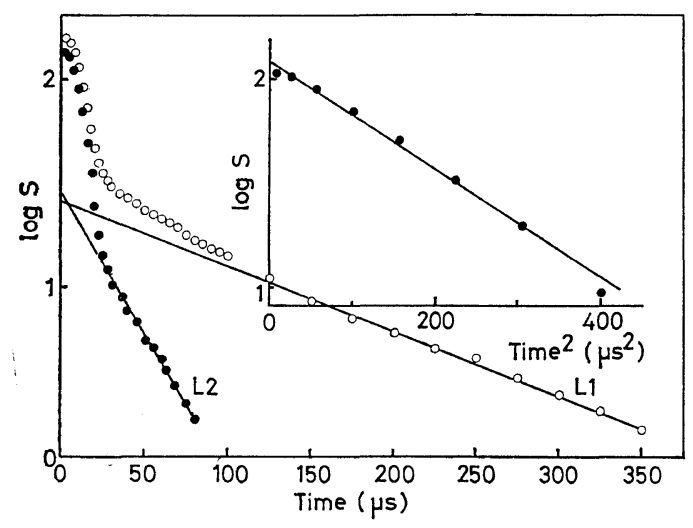

Figure 2. Analysis of the solid echo decay of the 2-8-MG at $25^{\circ} \mathrm{C}$. in the upper region of Figure 2 plotted against time squared. The Gaussian decay curve can be written $^{8}$ as

$$
S(t)=S_{0} \exp \left[-\left(t / T_{2}\right)^{2} / 2\right]
$$

It is concluded from the analysis of the solid echo decay that the decay with the shortest $T_{2}$ follows a Gaussian function and may be ascribed to the crystalline phase of the sample, and that the other decays, with the intermediate and longest $T_{2}$, follow an exponential function and may be ascribed to deays from the motionally narrowed region of the sample. All the solid echo decays obtained in this study were analyzed in this manner.

\section{Temperature Dependence of Spin-Spin Relaxation Times}

Figure 3 shows the temperature dependence of $T_{2}$ for the 2-8-MG and $10-16-\mathrm{MG}$. A 


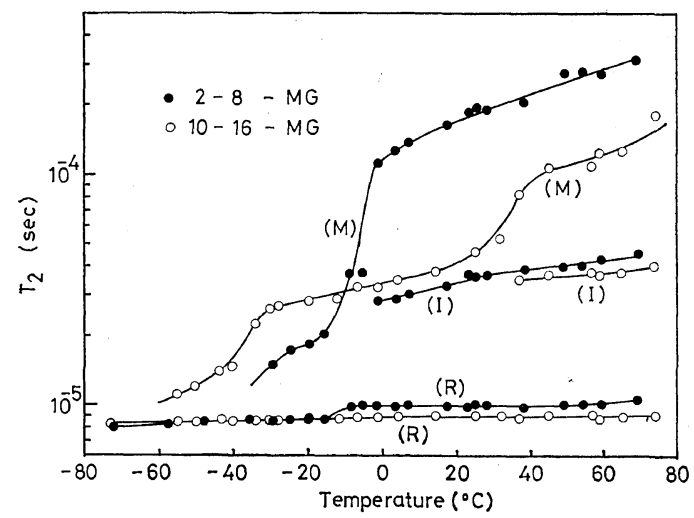

Figure 3. Temperature dependence of $T_{2}$ for the 2-8-MG and 10-16-MG. (M), (I), and (R) indicate mobile, intermediate, and rigid phases, respectively.

significant difference in the temperature dependence of $T_{2}$ is found between them. In the 10-16$\mathrm{MG}$, the shortest $T_{2}\left(T_{2 \mathrm{c}}\right)$ shows an almost constant value over the temperatures studied. The longest $T_{2}\left(T_{2 \mathrm{a}}\right)$ exhibits two transitions, at around -40 and $35^{\circ} \mathrm{C}$. At about $35^{\circ} \mathrm{C}$ a third component with an intermediately long $T_{2}\left(T_{2 \mathrm{~m}}\right)$ becomes apparent, but no distinct transition is found in this component. In the $2-8-\mathrm{MG}, T_{2 \mathrm{c}}$ is almost invariant from -70 to $70^{\circ} \mathrm{C}$, although a minor increase occurs between -20 and $0^{\circ} \mathrm{C}$. The $T_{2 a}$ component shows two abrupt increases, at around -30 and $-7^{\circ} \mathrm{C}$. At above $-3^{\circ} \mathrm{C} T_{2 \mathrm{~m}}$ becomes apparent, but no clear transition is found in it, just as in the case of the 10-16-MG. First, the temperature dependences of $T_{2 \mathrm{a}}$ for the two polyesters are considered. Both samples exhibit two obvious transitions in the $T_{2 \mathrm{a}}$ component. Correlation frequencies for the various motions responsible for the transitions were computed according to eq $3:{ }^{11}$

$$
\nu_{\mathrm{c}}=1 / 2 \pi T_{2 \mathrm{wt}}
$$

where $1 / T_{2 \mathrm{wt}}^{2}=1 / T_{2 l \mathrm{t}}^{2}-1 / T_{2 \mathrm{ht}}^{2}$, and the subscripts $l t$ and $h t$ refer, respectively, to the low and high temperature sides of the transition. The calculated values are compared with existing dielectric data ${ }^{4}$ in a transition map, shown in Figure 4. The low and high temperature transitions of the polyesters lie close to the $\gamma$ - and $\beta$-relaxations, respectively, at frequencies where the bulk of dielectric data

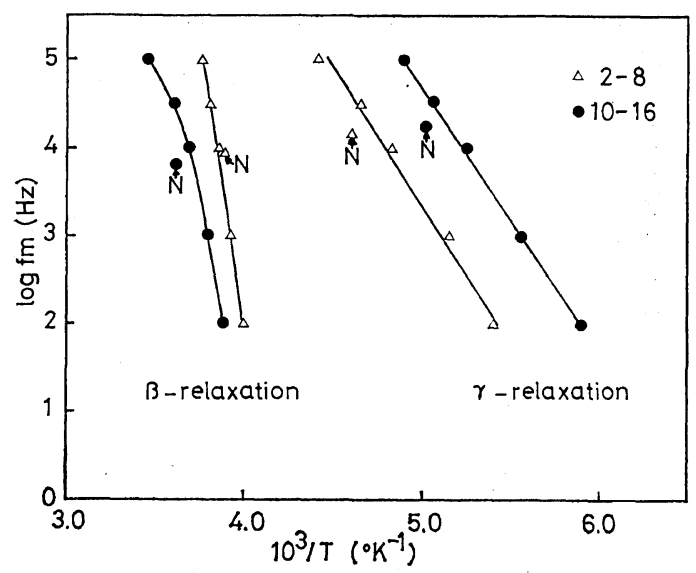

Figure 4. Comparison of the NMR correlation frequency data $(\mathrm{N})$ with dielectric results.

have been recorded. The $\gamma$ - and $\beta$-relaxations have been attributed to the local mode motion and micro-Brownian motion, respectively, in the noncrystalline region. ${ }^{4}$ Present NMR results also support these conclusions, since the $T_{2 \mathrm{a}}$ component airses from the noncrystalline regions.

The value of $T_{2 \mathrm{a}}$ of the 2-8-MG and 10-16MG on the high-temperature side of the $\gamma$-transition are 17 and $27 \mu \mathrm{sec}$, respectively. The expression for the $T_{2}$ depends on the model for molecular motion, but if we assume that the relaxation is due to reorientation of the dipole-dipole vector, we can then write ${ }^{10, p^{300}}$

$$
1 / T_{2}=(10 / 3) M_{2} \tau
$$

where $M_{2}$ is the second moment of the motional phase and $\tau$ is the reorientational correlation time of the dipole-dipole vector. Equation 4 has assumed that $\omega_{\mathrm{o}} \tau \gg 1$, where $\omega_{\mathrm{o}}$ is the nuclear precession angular velocity of $3.8 \times 10^{8} \mathrm{rad} / \mathrm{sec}$, and this assumption is valid when $T_{2} \ll T_{1}$. The spin-lattice relaxation times $\left(T_{1}\right)$ of these polyesters at the $\gamma$-relaxation is of the order of msec. Therefore the assumption mentioned above is valid. The concentration of hydrogen nuclei in the $10-16$ polyester is greater than in the $2-8$ polyester; therefore, the second moment of the 10-16 polyester is greater than that of the $2-8$ polyester and hence cannot explain the longer $T_{2 \mathrm{a}}$ found for the 10-16-MG. Evidently the difference in $T_{2 \mathrm{a}}$ is due to a difference in $\tau$ of the two polyesters. That is $\tau$ of the $10-16-\mathrm{MG}$ is shorter than that 
of the 2-8-MG. This result contradicts our expectation, deduced from the chain flexibility of the polymers, that the chain mobility of the $2-8$ MG would be larger than that of the 10-16-MG. As has been reported, ${ }^{4}$ the $\gamma$-transition of the polyesters is a local mode motion of main chains; such a relaxation is known to depend sensitively on the morphology. ${ }^{12,13}$ In order to elucidate the effects of morphology on the molecular motion, the temperature dependence of $T_{2}$ for single crystals of $2-8$ polyester ( $2-8-\mathrm{SG})$ was also investigated. The result is compared with that of melt-grown 2-8 polyester $(2-8-\mathrm{MG})$ in Figure $5 . \quad T_{2 \mathrm{a}}$ of

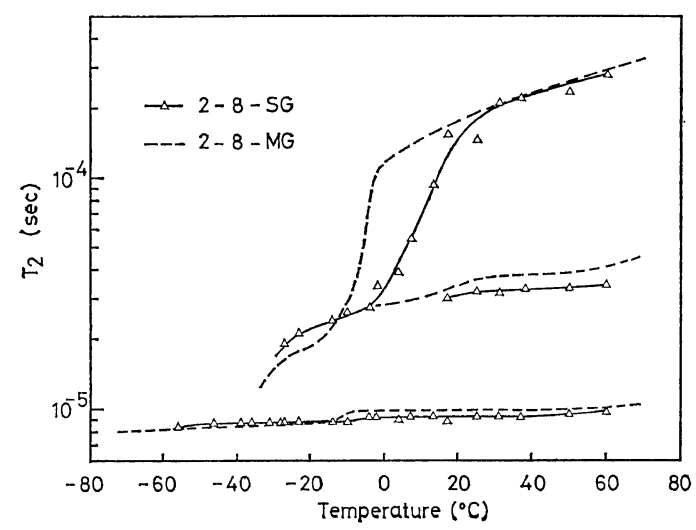

Figure 5. Temperature dependence of $T_{2}$ for the 2-8-SG.

the 2-8-SG on the high temperature side of the $\gamma$-transition is $22 \mu \mathrm{sec}$, which is a somewhat longer value than that of the $2-8-\mathrm{MG}$. On considering the information that polymer single crystals have a relatively more simple morphology than the melt-grown crystals, ${ }^{15}$ it seems that the simplification of morphology in the noncrystalline regions leads to a reduction of degree of restriction imposed upon the local mode motions of the polyesters. In a large scale motion such as microBrownian motion, the effect of interchain dipole attraction on the molecular mobility must be taken into account, ${ }^{4}$ but in the local mode motion, this effect is considered to be very small. These considerations suggest that the difference of $T_{2 \mathrm{a}}$ which is found between 2-8-MG and 10-16-MG may be caused by the difference of morphology in the noncrystalline region of the polyester, and also suggest that the greater mobility of the $10-16$ -
MG can be understood in terms of the much more simple morphology of the 10-16-MG, compared with that of the 2-8-MG.

In the $\beta$-transition, which corresponds to the glass transition of the polyesters, ${ }^{4}$ a rapid increase of $T_{2 \mathrm{a}}$ is found in both polyesters. The five-fold increase in $T_{2 \mathrm{a}}$ of the 2-8-MG over the two-fold increase of the same component in the 10-16MG tends to suggest that as the ester group concentration of the polyester is increased, the activation energy change on passing through the glass transition increases. Although the value of $T_{2 \mathrm{a}}$ on the high temperature side on the $\beta$-transition of the $2-8-\mathrm{MG}$ is quite similar to that of the 2-8-SG, this value is fairly large compared with that of $10-16-$ MG. We have reported ${ }^{4}$ that the glass transition temperature of the polyesters with rather low ester group concentrations is mainly controlled by chain flexibility, while the glass transition of the polyesters with high concentrations is shifted to higher temperatures, owing to interchain dipole attraction, rather than occurring at the expected temperature deduced from the chain flexibility. Present NMR results suggest that chain flexibility is the main factor, rather than the dipole attraction or morphology, needed to express the mobility of the amorphous segments on the high temperature side of the glass transition.

$T_{2 \mathrm{c}}$ of the 2-8-MG shows a small increase at around $-10^{\circ} \mathrm{C}$. However, this increase is less pronounced in the 2-8-SG sample and in the chemically treated high crystalline $2-8$ polyester (to be shown in a later section) and furthermore this increase is found through the $\beta$-transition. Therefore, the small increase of $T_{2 \mathrm{c}}$ of $2-8-\mathrm{MG}$ implies that, as the mobility in the amorphous segments is increased, the motions which the amorphous phase experiences on passing through the glass transition exert a larger influence on the motions of the crystalline component, probably at the boundaries of the two phases. It is of interest that this phenomenon is not found in the 10-16-MG.

The first appearance of $T_{2 \mathrm{~m}}$ for the 2-8-MG, $2-8-\mathrm{SG}$, and $10-16-\mathrm{MG}$ is around the $\beta$ transition temperature. The intermediate $T_{2}$ has been found in polyethylene and Nylon 6 by Fujimoto, et al., ${ }^{5}$ but the origin of this component has not been clarified. The present NMR results show that the intermediate phase can exist when 
the molecular motion of the amorphous phase is of the order of micro-Brownian motion and yet the molecular motion in the crystalline phase is not so active as to melt the crystalline state. It is thus of interest to investigate the nature of the intermediate phase of the polyesters.

Nature of the Intermediate Phase of the Polyester

Figure 6 shows the temperature dependence of the fraction of each phase, which was estimated from the value at the intersection with the vertical axis of each straight line obtained in the decomposition procedure at time $t=0$ for the 2-8-MG. The rigid fraction begins to decrease at about $-40^{\circ} \mathrm{C}$, almost levels off between -20 and $10^{\circ} \mathrm{C}$, and then decreases again. On the other hand, a very characteristic behavior is seen in the plot of mobile fraction against temperature. A sudden decrease of mobile fraction is found at around $0^{\circ} \mathrm{C}$, at which the intermediate phase is first observed. The intermediate fraction is almost invariant from 0 to $25^{\circ} \mathrm{C}$ and then decreases gradually as the temperature is raised further. However, the (mobile+intermediate) fraction increases almost linearly with increasing temperature, while at the same time the rigid fraction begins to decrease again. These facts suggest that the intermediate phase does not originate from the crystalline interior but from the restricted portion in the noncrystalline regions of the polymer. With regard to this

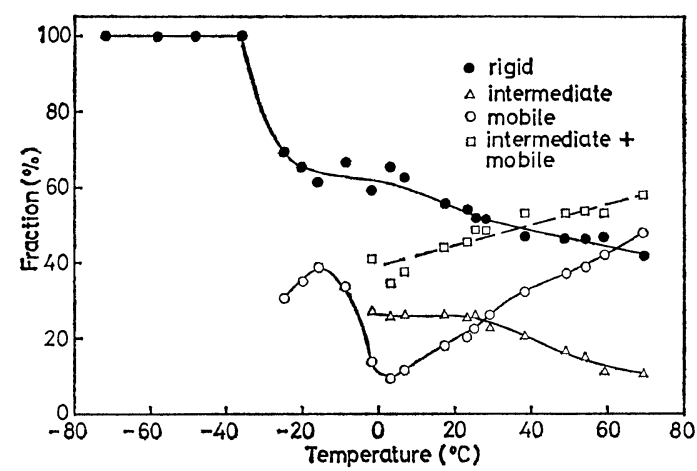

Figure 6. Temperature dependence of the fraction of the three phases for the $2-8-\mathrm{MG}$.

point, further information was obtained from the NMR data for the chemically degraded 2-8 polyester.

The dependence of crystallinity, molecular weight, long spacing, and melting peak temperature on the degradation time is shown in Figure 7. The crystallinity increases gradually with increasing reaction time, and levels off after $20 \mathrm{hr}$ of treatment. The highest crystallinity, viz., $85 \%$, is attained. On the other hand, the molecular weight and long spacing drop rapidly during the first few hours and then become nearly constant. These results directly indicate that the increase in crystallinity is due largely to solubilization and removal of noncrystalline segments and that the

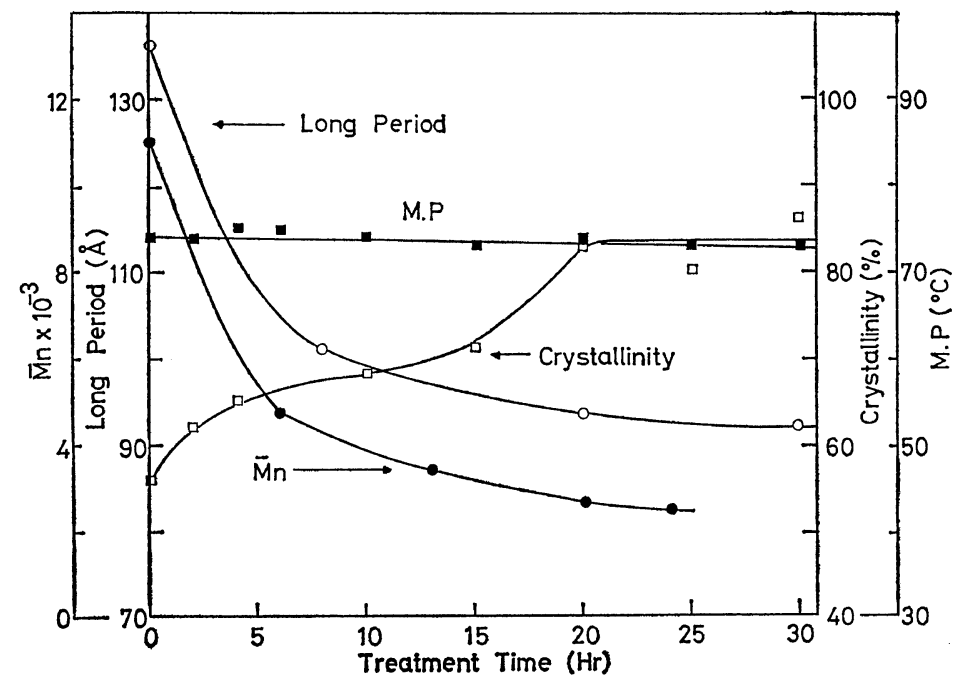

Figure 7. Degradation time dependence of crystallinity, molecular weight, long period, and melting temperature for the $2-8$ polyester. 
crystalline core is not destroyed by this treatment. The rapid decrease in molecular weight and the gradual increase in crystallinity suggest that no crystallization of low molecular weight materials which were eliminated from the noncrystalline regions occurs during the degradation.

The spin-spin relaxation times of $20 \mathrm{hr}$ degraded 2-8 polyester $(2-8-20 \mathrm{H})$ against temperature is shown in Figure 8. The following results are evident from this figure. First, the slight increase of $T_{2 \mathrm{c}}$ observed in the 2-8-MG disappears in the $2-8-20 \mathrm{H}$; second, $T_{2 \mathrm{a}}$ becomes more longer and does not show the $\gamma$-transition as a consequence of the degradation; and third, the temperature dependence of $T_{2 \mathrm{~m}}$ of the $2-8-20 \mathrm{H}$ is quite similar to that of the $2-8-\mathrm{MG}$.

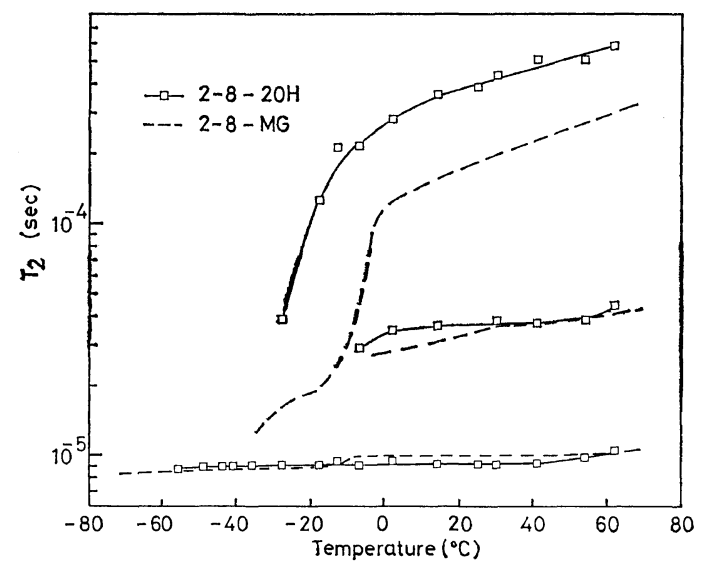

Figure 8. Temperature dependence of $T_{2}$ for the 2-8-20 H.

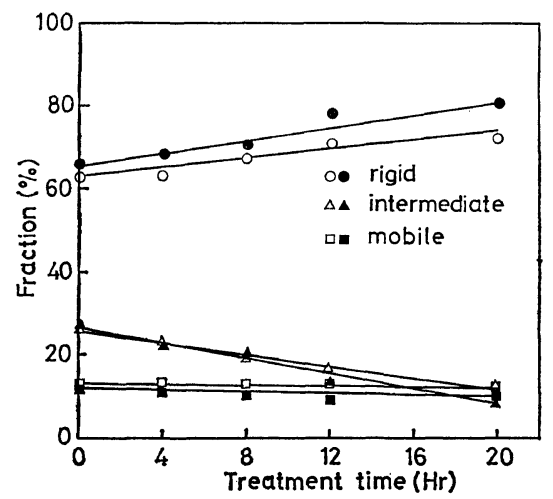

Figure 9. Degradation time dependence of the fraction of the three phases at $0^{\circ} \mathrm{C}(\boldsymbol{\Delta}, \Delta$, and $\mathbf{\square})$ and $10^{\circ} \mathrm{C}$ $(O, \square$, and $\triangle$ ).
The degradation time dependence of the fraction of each component is shown in Figure 9. As is shown in Figure 6, the fractions are sensitive to temperature, so the fractions plotted in Figure 9 were obtained in the temperature range where the amorphous segments undergo excited motions and the rigid fraction or the dynamical degree of crystallinity ${ }^{16}$ still maintains a constant value. The rigid fraction increases linearly with increasing degradation time; this result is consistent with the result obtained from the DSC measurements. The mobile component shows an almost constant value irrespective of increasing degradation time. This may not be unreasonable, since the removal of amorphous segments and the increase of end groups during the degradation would take place at the same time in the sample. This is supported by the fact that the mobility of mobile segments increases appreciably during a prolonged reaction. With increasing reaction time, the intermediate fraction decreases. As was mentioned, the degradation does not proceed to the crystalline region. Therefore, it seems that the increase of crystallinity results in an elimination of the intermediate component which appears in the noncrystalline regions of the polyester. The molecular mobility of the intermediate phase remains almost unaltered, despite the fairly large reduction in the amount of this fraction. Furthermore, its mobility is less sensitive to temperature, like that of the crystalline regions, and maintains a rather shorter value of $T_{2 \mathrm{~m}}$ than that of the mobile phase. These indicate the presence of fairly constrained segments in the intermediate phase. Fujimoto, et al., ${ }^{5}$ suggested in regard to the intermediate phase that it may exist in almost all crystalline polymers and seems to have no crystalline order as in the crystalline state, nor to be in perfect disorder as in the amorphous state.

Atkins, et al. ${ }^{1}$ have carried out an X-ray diffraction analysis of the lamellar structure of 66-Nylon single crystals. They found that sharp chain foldings occur at the lamellar surface, but that half the lamellar thickness is composed of a less-perfect crystalline core; the chain packing becomes gradually looser in going from the lamellar interior to the fold surface.

On the basis of these considerations, the origin of the intermediate component is considered to be as follows: the intermediate phase seems to exist 
in the noncrystalline regions of the polyester, but this phase does not seem to be a completely amorphous region but a fairly restricted region in the noncrystalline regions including those of partially ordered segments near the lamellar surface.

The fraction of intermediate phase of 2-8-SG and $10-16-\mathrm{MG}$ (not shown in this paper) was about 0.6 times as less as that of the 2-8-MG. Therefore, the amount of the intermediate phase may be cotrolled by the differences of the sample history or chemical structure of the polyester. As shown in Figures 3, 5, and 8, however, the molecular mobility in this phase is almost insensitive to these differences.

Acknowledgement. The authors wish to express their thanks to Professor Masatami Takeda for his continued interest and encouragement during this investigation.

\section{REFERENCES}

1. E. D. T. Atkins, A. Keller, D. M. Sadler, and H. H. Wills, J. Polym. Sci., Polym. Phys. Ed., 10, 863 (1972).
2. T. Kanamoto, K. Tanaka, and H. Nagai, ibid., Part A-2, 9, 2043 (1971).

3. T. Kanamoto, ibid., Polym. Phys. Ed., 12, 2535 (1974).

4. M. Ito, S. Nakatani, A. Gokan, and K. Tanaka, ibid., Polym. Phys. Ed., 15, 605 (1977).

5. K. Fujimoto, T. Nishi, and R. Kado, Polym. J., 3, 448 (1972).

6. V. J. McBrierty, Polymer, 15, 503 (1974).

7. E. M. Arnett, J. G. Miller, and A. R. Day, J. Am. Chem. Soc., 72, 5635 (1950).

8. J. G. Powles and J. H. Strange, Proc. Phys. Soc. (London), 82, 6 (1963).

9. T. Kanamoto, T. Maeda, H. Hirose, and K. Tanaka, Rep. Prog. Polym. Phys. Jpn., 19, 183 (1976).

10. A. Abragam, "The Principle of Nuclear Magnetism," Oxford University Press, London, 1961, p 44.

11. D. W. McCall, Natl. Bur. Stds., Spec. Publ., 310, 475 (1969).

12. M. Ito, T. Kanamoto, and K. Tanaka, J. Polym. Sci. Polym. Letters Ed., 14, 189 (1976).

13. M. Ito, T. Kanamoto, and K. Tanaka, Bull. Chem. Soc. Jpn., 50, 967 (1977).

14. P. H. Geil, "Polymer Single Crystals," Wiley, New York, 1963.

15. R. Chûjô, J. Phys. Soc. Jpn., 18, 124 (1963). 